\title{
ROBUST VIBRATION CONTROL OF WIND-EXCITED HIGHRISE BUILDING STRUCTURES
}

\author{
Nengmou WANG, Hojjat ADELI \\ Departments of Civil, Environmental, and Geodetic Engineering and Electrical and Computer Engineering, \\ The Ohio State University, 470 Hitchcock Hall, 2070 Neil Avenue, Columbus, Ohio 43210
}

Received 27 Nov 2014; accepted 26 Jun 2015

\begin{abstract}
A robust filtered sliding mode control (SMC) approach is presented for vibration control of wind-excited highrise building structures. Rather than using a Lyapunov-function based control design, an alternative way is provided to find the control force based on the equivalent control force principle to obtain the control force. A low pass filter is properly selected to remove the high-frequency components of the control force while retaining the structural stability. The performance of the proposed filtered SMC is evaluated by application to a wind-excited 76-story building benchmark problem equipped with an active tuned mass damper (ATMD) on the roof. Due to the elimination of high-frequency part of the control force, the structure, sensors, actuators, and dampers are all less excited, and consequently their response is reduced compared with the unfiltered SMC approach. In addition, the required control forces are reduced which means a reduction in the size of actuators, thus making their implementation more practical. It is shown the proposed method is more robust to structural stiffness uncertainties compared with the linear quadratic Gaussian (LQG) algorithm and another implementation of SMC.
\end{abstract}

Keywords: vibration, control, wind, structure.

\section{Introduction}

Active, semi-active and hybrid vibration control of structures subjected to extreme dynamic loading such as earthquake or wind has been an active area of research in recent years (Jiang, Adeli 2008a, 2008b; Adeli, Jiang 2009; Adeli, Kim 2009). Fisco and Adeli (2011a) present a review of active and semi-active vibration control of structures. Fisco and Adeli (2011b) present a review of hybrid control systems and control Strategies. More recently, El-Khoury and Adeli (2013) summarize recent Advances on vibration control of structures under dynamic loading.

The motivation of the research is to design a robust controller (Boutalis et al. 2013; Rigatos 2013; Tolu et al. 2013) with high performance for vibration control of large civil structures, a challenging research problem due to nonlinear structural behavior and many system uncertainties (unknown disturbance/excitation, sensor measurement noise, actuator dynamics, and modeling error between assumed system models and real systems) (Nigdeli, Boduroğlu 2013; Amini et al. 2013; Amini, Zabihi-Samani 2014; Su et al. 2014). The idea of sliding mode control (SMC) consists of two parts: (1) find a sliding surface (defined as a desired linear combination of system states such as displacement, velocity, and acceleration) to stabilize the controlled system, and (2) find a control force to drive the response trajectory into the sliding surface with an exponential speed in time (Utkin 1993). SMC is especially useful for variable structure systems (e.g., when stiffnesses vary during a dynamic event) because the sliding surface is independent of the control input and system uncertainties (Utkin 1993). SMC has been used for control of civil structures by Yang et al. (1995) and Wu and Yang (2004). Wu (2003) presents experimental verification of SMC for vibration control of a regular 3-story building structure using a shaking table.

The main advantage of the SMC is that it is invariant to external excitation such as wind and earthquake and the variation of system parameters (such as structural stiffness and damping) during the dynamic event. The structural uncertainties can be represented by a linear combination of the control forces. However, the chattering in SMC is generally a problem that needs to be resolved for better control. Recently, the authors presented a time-varying method for determining the sliding gain function in the SMC. Two alternative tuning algorithms were proposed. A $90 \%$ to $95 \%$ reduction of chattering was achieved for the algorithm used for systems with sensor dynamics only. Using the second algorithm, the chattering was reduced by $70 \%$ to $90 \%$ for systems with noise and/or disturbance, and by $25 \%$ to $50 \%$ for systems

Corresponding author: Hojjat Adeli

E-mail: Adeli.1@osu.edu 
with a combination of disturbance, noise, and unmodeled dynamics.

Since high frequency components of the control input do not impact controlled civil structures significantly (their frequencies are substantially lower), a low pass filter can remove the high frequency part of the control input determined by SMC. Use of a low pass filter results in a reduction of maximum control input which is significant for the size of actuators and real implementation. In this article, a filtered sliding mode control approach is presented to reduce the response of civil structures subjected to wind excitation. It is applied to a 76-story windexcited benchmark highrise building structure.

\section{Problem formulation}

\subsection{Reduced order model of physical wind excited structures}

The equation of the motion for a linear structure subjected to wind loading is (Adeli et al. 1978):

$$
M \ddot{x}+C \dot{x}+K \boldsymbol{x}=-\eta \boldsymbol{W}+\boldsymbol{B}_{s} \boldsymbol{u}
$$

where $\boldsymbol{x} \in \boldsymbol{R}^{p}$ is the displacement vector, $\boldsymbol{M}, \boldsymbol{C}, \boldsymbol{K} \in \boldsymbol{R}^{p \times p}$ are mass, damping, and stiffness matrices, respectively, $\mathrm{p}$ is the number of degrees of freedom of the structure, $\boldsymbol{W} \in \boldsymbol{R}^{p}$ is the vector of wind excitation, $\boldsymbol{\eta} \in \boldsymbol{R}^{p \times p}$ is a matrix of excitation influence representing the variation of the wind over the height of the structure, $\boldsymbol{u}(t) \in R^{q}$ is the control force vector assuming the structure has $q$ actuators, and $\boldsymbol{B}_{s}(\boldsymbol{x}, \dot{\boldsymbol{x}}) \in R^{p \times q}$ is the matrix related to positions of the control forces. For ease of controller design, Eqn (1) is rewritten as follows:

$$
\dot{\boldsymbol{X}}=\boldsymbol{A X}+\boldsymbol{B} \boldsymbol{u}+\boldsymbol{E} \boldsymbol{W},
$$

where $\boldsymbol{X}=\left[\boldsymbol{x}^{T}, \dot{\boldsymbol{x}}^{T}\right]^{T} \in \boldsymbol{R}^{2 p}$ is the structural states. $\boldsymbol{A}=\left[\begin{array}{cc}0 & \boldsymbol{I} \\ -\boldsymbol{M}^{-1} \boldsymbol{K} & -\boldsymbol{M}^{-1} \boldsymbol{C}\end{array}\right] \in \boldsymbol{R}^{2 p \times 2 p}$ is the matrix representing the properties of structural mass, stiffness, and damping, $\quad \boldsymbol{B}=\left[\begin{array}{c}0 \\ \boldsymbol{B}_{s}\end{array}\right] \in \boldsymbol{R}^{2 p}$ is the vector of control locations, and $\boldsymbol{E}=\left[\begin{array}{c}0 \\ -\boldsymbol{\eta}\end{array}\right] \in \boldsymbol{R}^{2 p \times p}$ is a matrix of wind excitation. It is common in the control field to reduce the number of degrees of freedom (DOF) for large systems in order: a) to avoid ill-conditioning of large matrices; and b) reduce the number of required actuators. Consequently, the size of the model is reduced using a state order reduction technique by keeping the dominant eigenvalues of the matrix $\boldsymbol{A}$ (the smallest ones) while removing unimportant eigenvalues (the largest ones) (Davison 1966). Eqn (2) is reduced to:

$$
\dot{x}_{r}=A_{r} x_{r}+B_{r} u+E_{r} W
$$

where $\boldsymbol{x}_{\boldsymbol{r}} \in \boldsymbol{R}^{n}$ (the states that maintain the first $n$ eigenvalues and eigenvectors of the matrix $\boldsymbol{A}, n<2 p$ ), $\boldsymbol{A}_{\boldsymbol{r}} \in \boldsymbol{R}^{n \times n}, \quad \boldsymbol{B}_{\boldsymbol{r}} \in \boldsymbol{R}^{n}, \boldsymbol{E}_{\boldsymbol{r}} \in \boldsymbol{R}^{n \times p}$. The measured states from sensors with noise are expressed as:

$$
\begin{gathered}
\boldsymbol{y}_{\boldsymbol{r}}=\boldsymbol{C}_{\boldsymbol{y} \boldsymbol{r}} \boldsymbol{x}_{\boldsymbol{r}}+\boldsymbol{D}_{\boldsymbol{y} \boldsymbol{r}} \boldsymbol{u}+\boldsymbol{F}_{\boldsymbol{y} \boldsymbol{r}} \boldsymbol{W}+\boldsymbol{v}_{\boldsymbol{r}}, \\
\boldsymbol{y}_{\boldsymbol{r}} \in \boldsymbol{R}^{r}, \quad \boldsymbol{C}_{\boldsymbol{y} \boldsymbol{r}} \in \boldsymbol{R}^{r \times n}, \quad \boldsymbol{D}_{\boldsymbol{y} \boldsymbol{r}}=0 \in \boldsymbol{R}^{r}, \quad \boldsymbol{F}_{\boldsymbol{y} \boldsymbol{r}} \in \boldsymbol{R}^{r \times p} .
\end{gathered}
$$

These matrices and vectors are obtained from the state order reduction technique. $\boldsymbol{v}_{\boldsymbol{r}} \in \boldsymbol{R}^{r}$ is assumed to be uncorrelated Gaussian white noise which is not measurable. The consideration of $\boldsymbol{v}_{\boldsymbol{r}}$ is due to the existence of sensor noise in practical situations.

Civil structures are generally assumed to be (1) stable: the structural response is reduced if the control force is properly calculated and applied, and (2) observable: if only part of the reduced states $\boldsymbol{x}_{\boldsymbol{r}}$ can be measured, $\boldsymbol{x}_{\boldsymbol{r}}$ can be determined by designing an observer to estimate the remaining states.

\subsection{Observer design}

Since the vector of states $\boldsymbol{x}_{\boldsymbol{r}}$ in Eqn (3) is only partially available by measurement, an observer is designed to estimate the system states as follows (Skelton, Ikeda 1989):

$$
\dot{\hat{x}}_{r}=A_{r} \hat{x}_{r}+B_{r} u+L\left(y_{r}-\hat{y}_{r}\right),
$$

where $\hat{\boldsymbol{y}}_{\boldsymbol{r}}=\boldsymbol{C}_{\boldsymbol{y} \boldsymbol{r}} \hat{\boldsymbol{x}}_{\boldsymbol{r}}+\boldsymbol{D}_{\boldsymbol{y} \boldsymbol{r}} \boldsymbol{u}$ and an observer gain matrix $\boldsymbol{L}$ is defined in the following form:

$$
\boldsymbol{L}=\left(\boldsymbol{P}_{0} \boldsymbol{C}_{\boldsymbol{y r}}^{\boldsymbol{T}}+\boldsymbol{S}_{0}\right) \boldsymbol{R}_{0}^{-1} .
$$

In Eqn (6), $\boldsymbol{P}_{0}$ is the solution of the Riccati equation $\boldsymbol{P}_{0} A_{0}+A_{0}^{\boldsymbol{T}} \boldsymbol{P}_{0}-\boldsymbol{P}_{0} \boldsymbol{C}_{\boldsymbol{y r}}^{\boldsymbol{T}} \boldsymbol{R}_{0}^{-1} \boldsymbol{C}_{\boldsymbol{y}} \boldsymbol{P}_{0}^{\boldsymbol{T}}+\boldsymbol{Q}_{0}-\boldsymbol{S}_{0} \boldsymbol{R}_{0}^{-1} \boldsymbol{S}_{0}^{\boldsymbol{T}}=0$ (Saleh, Adeli 1996, 1997; Adeli, Saleh 1999), where $\boldsymbol{A}_{0}=\boldsymbol{A}_{\boldsymbol{r}}-\boldsymbol{C}_{\boldsymbol{y}}^{\boldsymbol{T}} \boldsymbol{R}_{0}^{-1} \boldsymbol{S}_{0}^{\boldsymbol{T}}$, and the weight matrices $\boldsymbol{Q}_{0}, \boldsymbol{R}_{0}$ and $\boldsymbol{S}_{0}$ are given as follows (Skelton, Ikeda 1989):

$$
\begin{gathered}
Q_{0}=E_{r} S_{w w} E_{r}^{T}, \quad S_{0}=E_{r} S_{w w} F_{y r}^{T}, \\
R_{0}=S_{v_{r} v_{r}}+F_{y r} S_{w w} F_{y r}^{T} .
\end{gathered}
$$

In Eqn (7) $\boldsymbol{S}_{\boldsymbol{w} w}$ and $\boldsymbol{S}_{\boldsymbol{v}_{\boldsymbol{r}} \boldsymbol{v}_{\boldsymbol{r}}}$ are power spectral density matrices of $\boldsymbol{W}$ and $\boldsymbol{v}_{\boldsymbol{r}}$, respectively, which can be specified by the control algorithm designer. A vector of state errors is defined as $\boldsymbol{e}=\boldsymbol{x}_{\boldsymbol{r}}-\hat{\boldsymbol{x}}_{\boldsymbol{r}}$. The derivative of this error vector is found by subtracting Eqn (5) from Eqn (3):

$$
\dot{e}=\left(A_{r}-L C_{y r}\right) e+E_{r} W-L\left(F_{y r} W+v_{r}\right) .
$$

Since $\boldsymbol{W}$ and $\boldsymbol{v}_{\boldsymbol{r}}$ are unknown terms, it is necessary to assume that $\boldsymbol{E}_{\boldsymbol{r}} \boldsymbol{W}-\boldsymbol{L}\left(\boldsymbol{F}_{\boldsymbol{y} r} \boldsymbol{W}+\boldsymbol{v}_{\boldsymbol{r}}\right)$ is small enough to have an insignificant influence on the convergence of the error vector $\boldsymbol{e}$. Matrix $\boldsymbol{L}$ defined by Eqn (6) results in all 
the eigenvalues of $\left(\boldsymbol{A}_{\boldsymbol{r}}-\boldsymbol{L} \boldsymbol{C}_{\boldsymbol{y r}}\right)$ to have a negative real part. This in turn provides system stability and ensures that error vector $\boldsymbol{e}$ will converge to zero, i.e., $\hat{\boldsymbol{x}}_{\boldsymbol{r}}$ will reach its desired value in a finite time as well.

\section{Filtered sliding mode control}

\subsection{Controller design}

In SMC a sliding surface vector $\boldsymbol{s}$ is designed first to stabilize the controlled system followed by determination of control forces to drive the response trajectory into the discontinuous sliding surfaces. Substituting Eqn (4) into (5) and noting that $\boldsymbol{D}_{\boldsymbol{y} \boldsymbol{r}}=0$ (because the vector of measured states $\boldsymbol{y}_{\boldsymbol{r}}$ is not related to the control force) yields:

$$
\dot{\hat{\boldsymbol{x}}}_{r}=A_{r} \hat{\boldsymbol{x}}_{r}+B_{r} \boldsymbol{u}+E_{r} \gamma,
$$

where $\boldsymbol{E}_{\boldsymbol{r}}=\left[\boldsymbol{L} \boldsymbol{C}_{\boldsymbol{y}}, \boldsymbol{L} \boldsymbol{F}_{\boldsymbol{y}}, \boldsymbol{L}\right]$, and $\tilde{\boldsymbol{a}}^{\boldsymbol{T}}=\left[\boldsymbol{e}, \boldsymbol{W}, \boldsymbol{v}_{\boldsymbol{r}}\right]$. Wu and Yang (2004) use a modified SMC approach where they find the control force based on a Lyapunov function. In this article, an alternative method is provided to find the control force based on the equivalent control force principle (Utkin 1993). Estimated states are divided into two parts, without actuators (control forces) $\left(\dot{\hat{x}}_{1}\right)$ and with actuators (control forces) ( $\left.\dot{\hat{\boldsymbol{x}}}_{2}\right)$ and Eqn (9) is transformed to the following two equations:

$$
\begin{gathered}
\dot{\hat{\boldsymbol{x}}}_{1}=\boldsymbol{A}_{11} \hat{\boldsymbol{x}}_{1}+\boldsymbol{A}_{12} \hat{\boldsymbol{x}}_{2}+\boldsymbol{E}_{\boldsymbol{r} 1} \gamma ; \\
\dot{\hat{\boldsymbol{x}}}_{2}=\boldsymbol{A}_{21} \hat{\boldsymbol{x}}_{1}+\boldsymbol{A}_{22} \hat{\boldsymbol{x}}_{2}+\boldsymbol{B}_{2} \boldsymbol{u}+\boldsymbol{E}_{\boldsymbol{r} 2} \gamma,
\end{gathered}
$$

where $\hat{\boldsymbol{x}}_{\boldsymbol{r}}=\left[\begin{array}{l}\hat{\boldsymbol{x}}_{1} \\ \hat{\boldsymbol{x}}_{2}\end{array}\right], \quad \hat{\boldsymbol{x}}_{1} \in \boldsymbol{R}^{n-m}, \quad \hat{\boldsymbol{x}}_{2} \in \boldsymbol{R}^{m}, m$ is the number of actuators, $\boldsymbol{A}_{\boldsymbol{r}}=\left[\begin{array}{ll}\boldsymbol{A}_{11} & \boldsymbol{A}_{12} \\ \boldsymbol{A}_{21} & \boldsymbol{A}_{22}\end{array}\right], \quad \boldsymbol{B}_{\boldsymbol{r}}=\left[\begin{array}{c}0 \\ \boldsymbol{B}_{2}\end{array}\right]$, $\boldsymbol{E}_{\boldsymbol{r}}=\left[\begin{array}{c}\boldsymbol{E}_{\boldsymbol{r} 1} \\ \boldsymbol{E}_{\boldsymbol{r} 2}\end{array}\right]$, and $\boldsymbol{A}_{11}, \boldsymbol{A}_{12}, \boldsymbol{A}_{21}, \boldsymbol{A}_{22}, \boldsymbol{B}_{2}, \boldsymbol{E}_{\boldsymbol{r} 1}$, and

$\boldsymbol{E}_{\boldsymbol{r} 2}$ are matrices of corresponding dimensions. It should be noted that $\operatorname{det}\left(\boldsymbol{B}_{2}\right) \neq 0$. The design of sliding mode control consists of two steps.

First, $\hat{\boldsymbol{x}}_{2}$ in Eqn (10) is treated similar to a control force and Eqn (10) is solved like an optimal control problem using a linear-quadratic method. Assume $\hat{\boldsymbol{x}}_{2}$ can be related to $\hat{\boldsymbol{x}}_{1}$ as:

$$
\hat{\boldsymbol{x}}_{2}=-\boldsymbol{P}_{1} \hat{\boldsymbol{x}}_{1}
$$

where $\boldsymbol{P}_{1} \in \boldsymbol{R}^{m \times(n-m)}$ is a gain matrix obtained by solution of a Riccati equation similar to the previous equation. Then, based on Eqn (12), the equation of the sliding surface is chosen as:

$$
\boldsymbol{s}=\boldsymbol{P}_{1} \hat{\boldsymbol{x}}_{1}+\hat{\boldsymbol{x}}_{2}=\boldsymbol{P} \hat{\boldsymbol{x}}_{\boldsymbol{r}},
$$

where $\boldsymbol{P}=\left[\boldsymbol{P}_{1}, \boldsymbol{I}\right]$ for ease of notation. The goal in SMC is to achieve $\boldsymbol{s}=0$.
The second step is to find control forces such that the response trajectory will always remain along sliding surfaces $\boldsymbol{s}=0$. Taking the derivative of $\boldsymbol{s}$ in Eqn (13) and using Eqn (9) yields:

$$
\dot{s}=P \dot{\hat{x}}_{r}=P\left(A_{r} \hat{x}_{r}+B_{r} u+E \gamma\right)=P A_{r} \hat{x}_{r}+P E_{r} \gamma+P B_{r} u .
$$

The inverse term $\left(\boldsymbol{P} \boldsymbol{B}_{\boldsymbol{r}}\right)^{-1}$ exists because of the existing of $\boldsymbol{B}_{r}{ }^{-1}$. Then, the following discontinuous equivalent control force is chosen for $\boldsymbol{s}$ to converge to zero:

$$
\boldsymbol{u}=\left(\boldsymbol{P} \boldsymbol{B}_{\boldsymbol{r}}\right)^{-1}\left[-\boldsymbol{P} \boldsymbol{A}_{\boldsymbol{r}} \hat{\boldsymbol{x}}_{\boldsymbol{r}}-\operatorname{Msign}(\boldsymbol{s})\right]
$$

where $M>\boldsymbol{P} \boldsymbol{E}_{\boldsymbol{r}} \boldsymbol{\gamma}$ is a time-varying gain chosen to be equal to $M=a \boldsymbol{s}+\delta$ ( $a$ and $\delta$ are positive constants), $\operatorname{sign}(\boldsymbol{s})=\left[\begin{array}{c}\operatorname{sign}\left(s_{1}\right) \\ \vdots \\ \operatorname{sign}\left(s_{m}\right)\end{array}\right]$. It should be noted that white noise in continuous time has infinite variance and cannot be sampled. Discrete-time white noise, on the other hand, has a finite variance with a constant power spectrum. Also, note that the observation noise sequence is obtained as a result of sampling the sensor outputs. Noise in this model is discrete and consequently has finite values, which implies the existing of $M$. In this research a non-constant $M$ is chosen resulting in the inequality $\delta<M$ which means the undesirable chattering in SMC is reduced. Substituting Eqn (15) into (14) yields:

$$
\dot{\boldsymbol{s}}=\boldsymbol{P} \boldsymbol{E}_{\boldsymbol{r}} \gamma-\operatorname{Msign}(\boldsymbol{s})
$$

Since $M>\boldsymbol{P} \boldsymbol{E}_{\boldsymbol{r}} \boldsymbol{\gamma},-\operatorname{Msign}(\boldsymbol{s})$ rather than $\boldsymbol{P} \boldsymbol{E}_{r} \gamma$ is the determining term in Eqn (16), which ensures that $\operatorname{sign}(\dot{\boldsymbol{s}})$ is always opposite of $\operatorname{sign}(\boldsymbol{s})$. As a result, the condition $s=0$ for the sliding surfaces will be achieved in a finite time, which means the system states will decay with an exponential speed in accordance with Eqn (12).

\subsection{First order low pass filter for the control force}

The control force proposed in Eqn (15) generally contains high-frequency components due to fast and frequent switching of the sliding mode control force. The high-frequency components have an insignificant influence on the system response since most civil structures have a low frequency compared with that of control force and it is unlikely that a resonant phenomenon will happen (Adeli, Kim 2004; Kim, Adeli 2004, 2005). Removing the high frequency components of the control force, however, results in a smaller force and actuator size without any loss of response reduction. In this research, a first order low pass filter is properly selected to remove the highfrequency components of the control force in Eqn (15) while maintaining structural stability. A filtered control force $\boldsymbol{u}_{f}$ is selected to satisfy: 


$$
\tau \dot{\boldsymbol{u}}_{f}+\boldsymbol{u}_{\boldsymbol{f}}=\boldsymbol{u}
$$

where the time constant $\tau \ll 1, \tau \gg T_{s}$ and $T_{S}$ is the sampling time interval. It is necessary to choose a proper $\tau$ that is not too large to cause an unstable situation for the controlled structure. The controlled structure will fall into unstable status if $\tau$ is too big (for example, when $\tau$ is close to 1). The stability is guaranteed by selection of a $\tau$ value such that structural response is always reduced. After applying a Laplace transformation to Eqn (17) the filtered control force $\boldsymbol{u}_{f}$ can be rewritten as:

$$
\boldsymbol{u}_{f}=\frac{\boldsymbol{u}}{\tau \Omega+1}=\frac{\left(\boldsymbol{P} \boldsymbol{B}_{\boldsymbol{r}}\right)^{-1}\left[-\boldsymbol{P} \boldsymbol{A}_{\boldsymbol{r}} \hat{\boldsymbol{x}}_{\boldsymbol{r}}-\operatorname{Msign}(\boldsymbol{s})\right]}{\tau \Omega+1},
$$

where $\Omega$ is a variable in the frequency domain.

\section{Example}

The filtered SMC method presented in this paper is applied to a benchmark control problem developed based on a 76-story, 306-m office tower proposed for the city of Melbourne, Australia (Yang et al. 2004). This reinforced concrete building consists of a central concrete core and an external concrete frame. The 153,000-metric ton slender building is sensitive to wind since its height-to-width ratio is 7.3 . It is modeled as a vertical cantilever structure with rigid floors and 76 degree-of-freedom (DOF) for the translational vibration as shown in Figure 1 (one DOF per floor). The plan view of this building is shown in Figure 2. The time histories of wind excitations for the $30^{\text {th }}, 50^{\text {th }}, 70^{\text {th }}$, and $76^{\text {th }}$ floor are shown in Figure 3 . Figure 4 shows a schematic architecture for vibration control

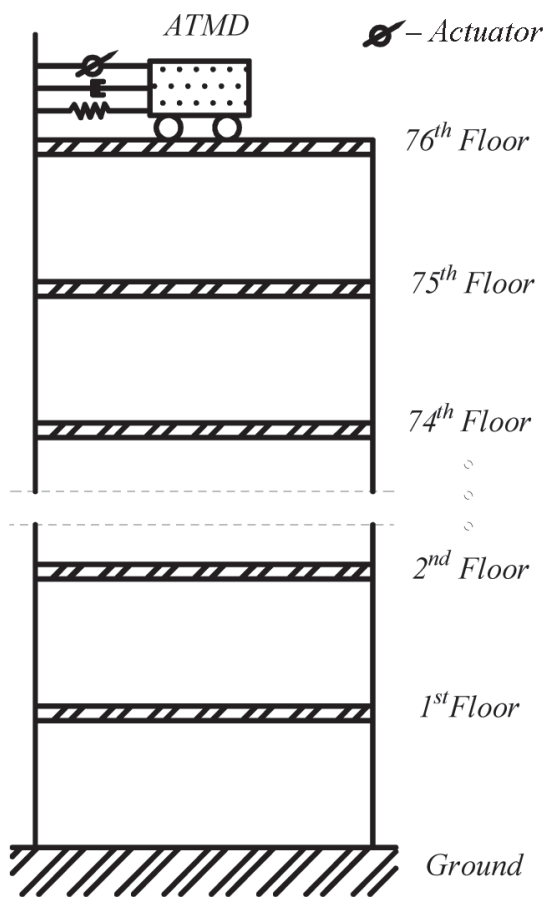

Fig. 1. Two-dimensional model of the 76-story highrise building

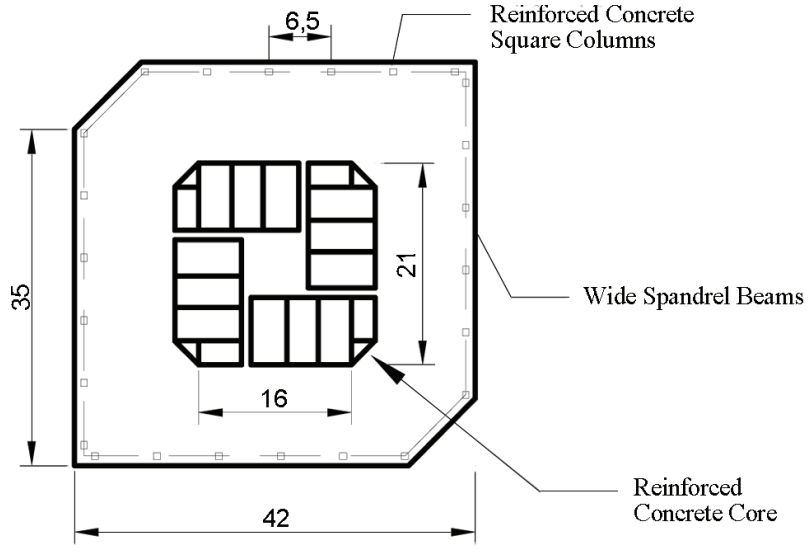

Fig. 2. Plan view of the highrise building (Unit: meter)

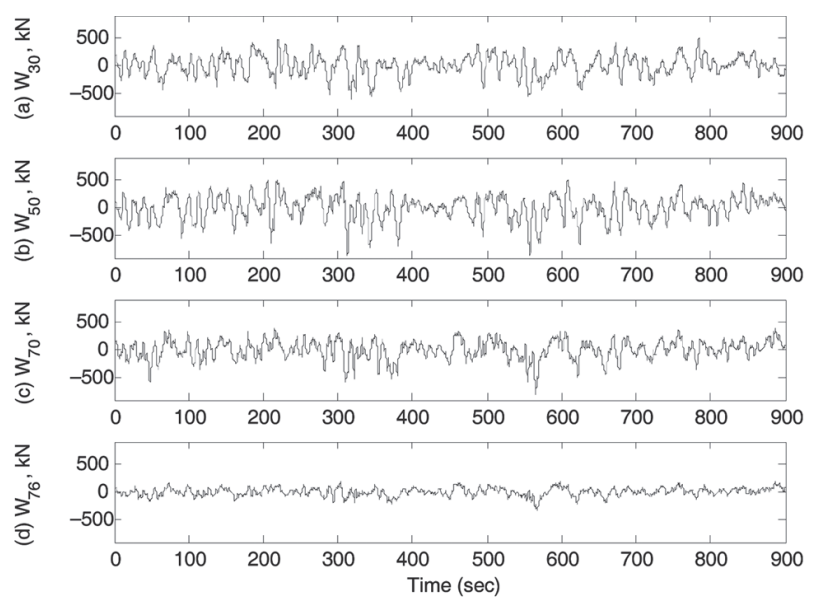

Fig. 3. Time histories of wind excitations for the $30^{\text {th }}, 50^{\text {th }}$, $70^{\text {th }}$, and $76^{\text {th }}$ floors

of the 76-story building using the approach presented in this paper.

The building is equipped with an active tuned mass damper (ATMD) (Amini et al. 2013) on the top floor. The equation of motion of the ATMD is (Smith, Coull 1992):

$$
m\left(\ddot{x}_{76}+\ddot{x}_{m}\right)+c_{m} \dot{x}_{m}+k_{m} x_{m}=u .
$$

Please refer to Appendix I for a detailed explanation of Eqn (19).

A reduced order system is constructed as follows: The state vector is $\boldsymbol{x}_{\boldsymbol{r}}=\left[x_{16}, x_{30}, x_{46}, x_{60}, x_{76}, x_{m}\right.$, $\left.\dot{x}_{16}, \dot{x}_{30}, \dot{x}_{46}, \dot{x}_{60}, \dot{x}_{76}, \dot{x}_{m}\right]^{T} \in \boldsymbol{R}^{12}$, the measured output vector is $\boldsymbol{y}_{\boldsymbol{r}}=\left[\ddot{x}_{50}, \ddot{x}_{76}, \ddot{x}_{m}\right]^{T}, \boldsymbol{A}_{\boldsymbol{r}} \in \boldsymbol{R}^{12 \times 12}, \boldsymbol{B}_{\boldsymbol{r}} \in \boldsymbol{R}^{12}$, $\boldsymbol{E}_{\boldsymbol{r}} \in \boldsymbol{R}^{12 \times 77}, \boldsymbol{C}_{\boldsymbol{y} r} \in \boldsymbol{R}^{3 \times 12}, \boldsymbol{D}_{\boldsymbol{y} r}=0 \in \boldsymbol{R}^{3}, \boldsymbol{F}_{\boldsymbol{y} r} \in \boldsymbol{R}^{3 \times 77}$, and $\boldsymbol{v}_{\boldsymbol{r}} \in \boldsymbol{R}^{3}$. Yang et al. (2004) show that the peak and RMS values of the structural response of the reduced order system are very close to that of the full order system. For the sake of comparison, the parameters used in the example are the same as those used by Yang et al. (2004) 


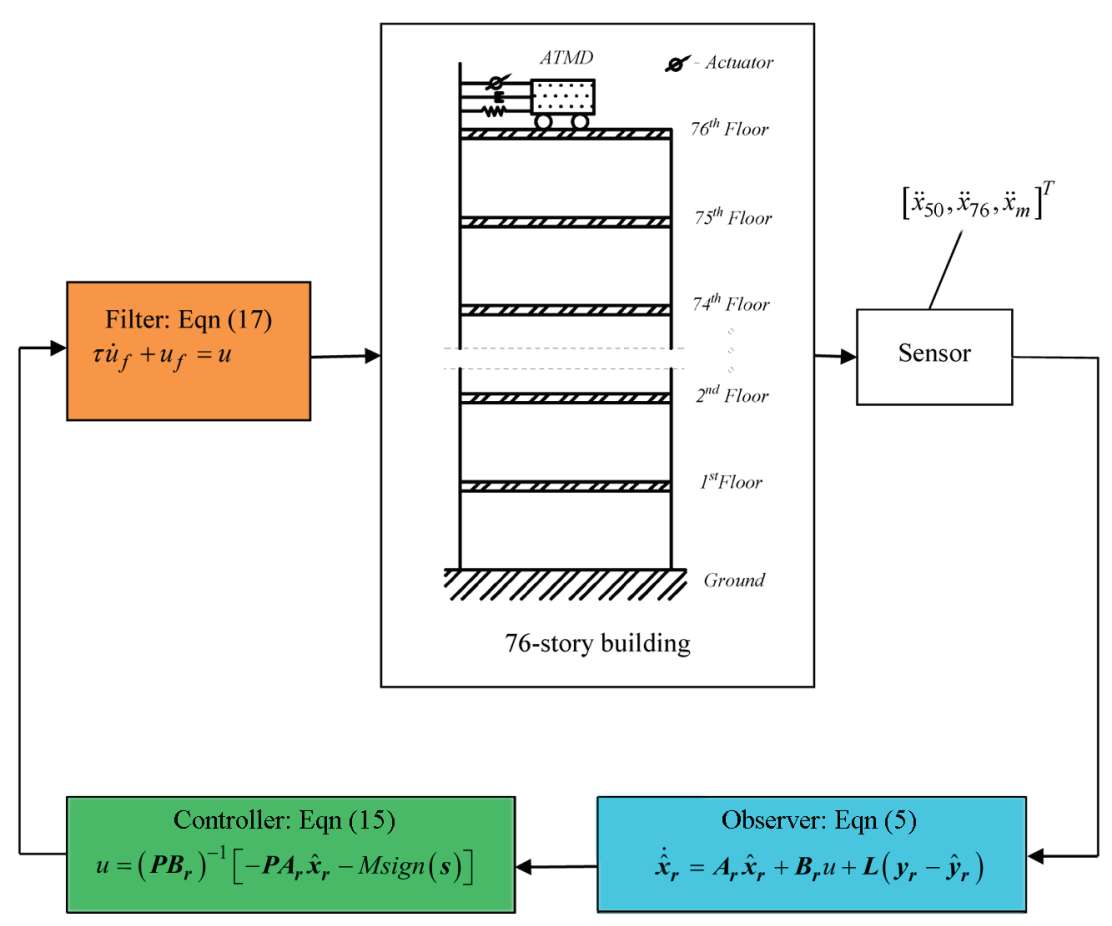

Fig. 4. Schematic architecture for vibration control of the 76-story building

as described in Appendix I. Other different parameters are described below.

The time constant in Eqn (17), $\tau=0.1$, is chosen by trial-and-error to avoid an unstable situation. As a general guideline, an initial value of 0.3 for $\tau$ is large enough. Then, this value is reduced iteratively with a step length of 0.05 until the performance cannot be improved in terms of the performance criteria. A value of $\delta=0.001$ is chosen by trial-and-error. The sampling time is $T_{S}=0.001 \mathrm{sec}$. Three different values are chosen for the constant $a=1.065,1.265$, and 1.865: the first two are chosen for comparison with LQG presented in Yang et al. (2004) and the SMC approach presented by Wu and Yang (2004), respectively. The last value is chosen to show that the structural vibrations can be reduced significantly. For the sake of comparison, only one actuator is used on the top floor of the structure similar to Yang et al. (2004).

Yang et al. (2004) defined 16 criteria for the benchmark problem summarized in the Appendix II. The lower the value of each criterion the more effective the control algorithm. Table 1 shows a comparison of 16 evaluation criteria for the proposed filtered SMC method with the LQG algorithm presented in Yang et al. (2004) as well as the unfiltered SMC using $a=1.065$. To test the robustness of the proposed method, a $\pm 15 \%$ stiffness uncertainty is applied in the simulation. It is found that criteria $J_{1}$ to $J_{4}$ and $J_{7}$ to $J_{10}$ of unfiltered SMC are slightly smaller than those of the LQG control, but the other 8 criteria are slightly higher for the unfiltered SMC. Therefore, in general the performance of the unfiltered SMC approach is similar to that of LQG control for vibration control of this building. Next, for the filtered SMC, it is found that most of the criteria are much or slightly smaller than that of both unfiltered SMC and LQG control, especially in maximum control force and actuator displacement which are significant due to the limitation of commercially available actuators. Also, the proposed filtered SMC with $\pm 15 \%$ stiffness uncertainty is less sensitive compared with the LQG algorithm.

Table 2 shows a comparison of the same 16 evaluation criteria for the proposed filtered SMC method with the SMC technique presented in $\mathrm{Wu}$ and Yang (2004) as well as the unfiltered SMC using 1.265. This table shows that the performance of unfiltered SMC is similar to that of Wu and Yang (2004). Without stiffness uncertainty, performance of the proposed filtered SMC in 12 criteria is better compared with the unfiltered SMC and the SMC method of Wu and Yang (2004). For all three different stiffness values the maximum control force of filtered SMC is less than that for the SMC method of Wu and Yang (2004). For the $\pm 15 \%$ stiffness uncertainty cases, the performance of filtered SMC is better than the other two control approaches with the exception of the values of $J_{7}$ and $J_{8}$ for the $+15 \%$ stiffness uncertainty case which are slightly higher than the corresponding values in Wu and Yang (2004).

The time histories of the structural response of the $75^{\text {th }}$ floor and control force on the top of the structure are shown in Figures 5 and 6 for filtered SMC with $a=1.865$ and $a=1.265$, respectively. It is found that the response has been reduced by using the filtered SMC compared with the case without control. Structural vibrations can be reduced significantly by choosing a proper value for the parameter a. 
Table 1. A comparison of evaluation criteria with LQG

\begin{tabular}{|c|c|c|c|c|c|c|c|c|c|c|c|}
\hline \multicolumn{4}{|c|}{ Filter SMC $(\alpha=1.065, \tau=0.1)$} & \multicolumn{4}{|c|}{ Unfiltered SMC ( $\alpha=1.065)$} & \multicolumn{4}{|c|}{ LQG (Yang et al. 2004) } \\
\hline 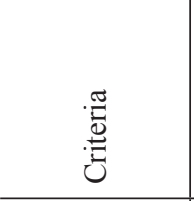 & \begin{tabular}{l}
0 \\
$\|$ \\
\multirow{y}{y}{}
\end{tabular} & $\begin{array}{l}\stackrel{0}{i n} \\
\stackrel{y}{\sharp} \\
y\end{array}$ & $\begin{array}{l}\stackrel{0}{i} \\
\stackrel{1}{1} \\
\| \\
y \\
y\end{array}$ & 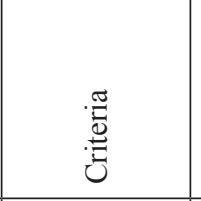 & $\begin{array}{l}0 \\
\| \\
\searrow \\
y\end{array}$ & $\begin{array}{l}\stackrel{0}{i n} \\
\| 1 \\
i\end{array}$ & $\begin{array}{l}\stackrel{0}{i} \\
\frac{1}{1} \\
\| \\
\grave{y}\end{array}$ & 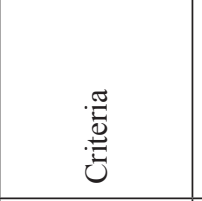 & $\begin{array}{l}0 \\
\| \\
\searrow \\
\searrow\end{array}$ & $\begin{array}{l}\stackrel{0}{i} \\
\| 1 \\
y\end{array}$ & 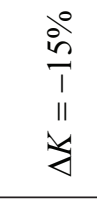 \\
\hline$J_{1}$ & 0.372 & 0.379 & 0.381 & $J_{1}$ & 0.367 & 0.362 & 0.385 & $J_{1}$ & 0.369 & 0.365 & 0.387 \\
\hline$J_{2}$ & 0.420 & 0.422 & 0.431 & $J_{2}$ & 0.415 & 0.407 & 0.435 & $J_{2}$ & 0.417 & 0.409 & 0.438 \\
\hline$J_{3}$ & 0.578 & 0.492 & 0.703 & $J_{3}$ & 0.577 & 0.486 & 0.709 & $J_{3}$ & 0.578 & 0.487 & 0.711 \\
\hline$J_{4}$ & 0.580 & 0.494 & 0.705 & $J_{4}$ & 0.579 & 0.488 & 0.711 & $J_{4}$ & 0.580 & 0.489 & 0.712 \\
\hline$J_{5}$ & 2.243 & 1.808 & 2.658 & $J_{5}$ & 2.297 & 1.836 & 2.746 & $J_{5}$ & 2.271 & 1.812 & 2.709 \\
\hline$J_{6}$ & 11.594 & 8.480 & 15.914 & $J_{6}$ & 12.399 & 8.786 & 17.198 & $J_{6}$ & 11.99 & 8.463 & 16.61 \\
\hline$\sigma_{u(\mathrm{kN})}$ & 34.15 & 28.49 & 44.25 & $\sigma_{u}(\mathrm{kN})$ & 34.96 & 29.09 & 45.51 & $\sigma_{u}(\mathrm{kN})$ & 34.07 & 28.29 & 44.32 \\
\hline$\sigma_{x_{m}(\mathrm{~cm})}$ & 22.74 & 18.32 & 26.95 & $\sigma_{x_{m}}(\mathrm{~cm})$ & 23.29 & 18.61 & 27.83 & $\sigma_{x_{m}}(\mathrm{~cm})$ & 23.03 & 18.37 & 27.46 \\
\hline$J_{7}$ & 0.382 & 0.434 & 0.482 & $J_{7}$ & 0.379 & 0.407 & 0.485 & $J_{7}$ & 0.381 & 0.411 & 0.488 \\
\hline$J_{8}$ & 0.431 & 0.457 & 0.534 & $J_{8}$ & 0.432 & 0.442 & 0.537 & $J_{8}$ & 0.432 & 0.443 & 0.539 \\
\hline$J_{9}$ & 0.710 & 0.617 & 0.781 & $J_{9}$ & 0.716 & 0.608 & 0.766 & $J_{9}$ & 0.717 & 0.607 & 0.770 \\
\hline$J_{10}$ & 0.718 & 0.624 & 0.791 & $J_{10}$ & 0.724 & 0.615 & 0.775 & $J_{10}$ & 0.725 & 0.614 & 0.779 \\
\hline$J_{11}$ & 2.244 & 1.875 & 2.846 & $J_{11}$ & 2.320 & 1.870 & 2.865 & $J_{11}$ & 2.300 & 1.852 & 2.836 \\
\hline$J_{12}$ & 66.690 & 53.326 & 117.713 & $J_{12}$ & 73.920 & 54.241 & 121.864 & $J_{12}$ & 71.96 & 52.68 & 118.33 \\
\hline $\max |u|(\mathrm{kN})$ & 118.44 & 107.15 & 167.09 & $\max |u|(\mathrm{kN})$ & 122.38 & 109.15 & 168.74 & $\max |u|(\mathrm{kN})$ & 118.24 & 105.58 & 164.33 \\
\hline $\max \left|x_{m}\right|(\mathrm{cm}) \mid$ & 72.48 & 60.55 & 91.93 & $\max \left|x_{m}\right|(\mathrm{cm})$ & 74.94 & 60.39 & 92.53 & $\max \left|x_{m}\right|(\mathrm{cm})$ & 74.29 & 59.83 & 91.60 \\
\hline
\end{tabular}

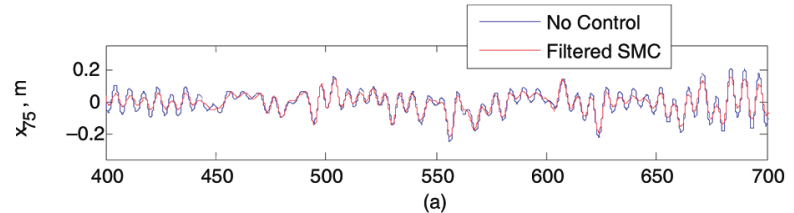

(a)
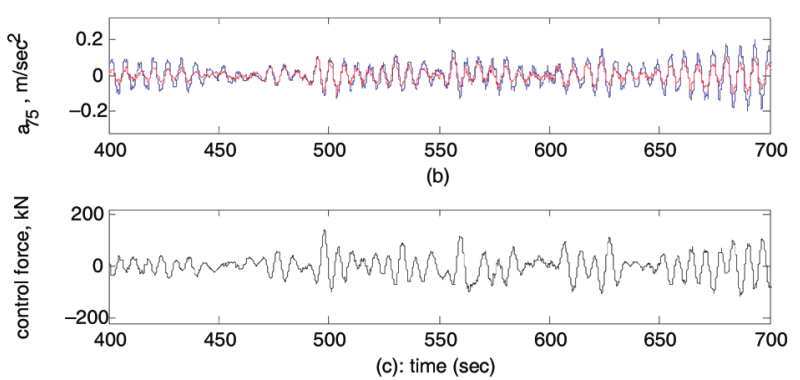

Fig. 5. Time series of structural response and control force for filtered SMC with $\alpha=1.865$ : (a) $\mathrm{x}_{75}$; (b) $\ddot{\mathrm{x}}_{75}$; and (c) control force

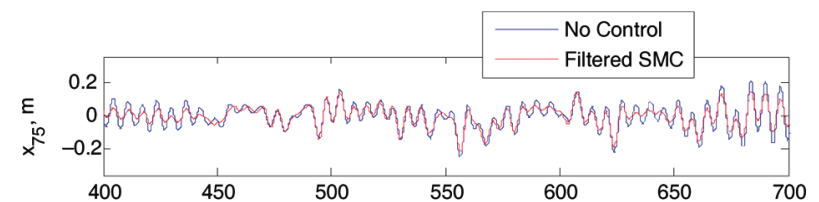

(a)

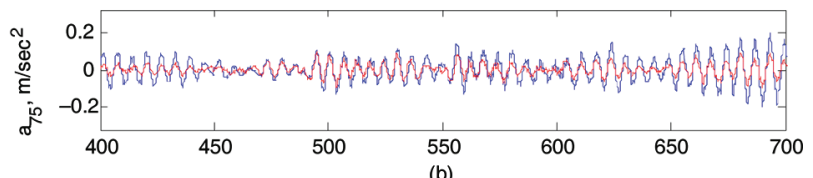
(b)

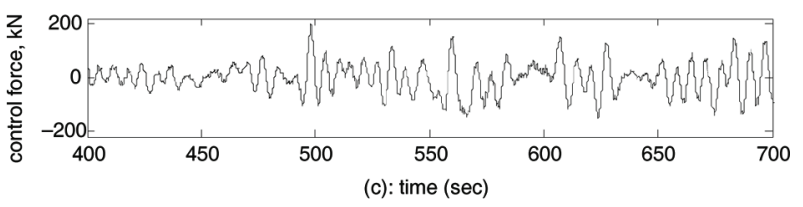

Fig. 6. Time series of structural response and control force using filtered SMC with $\alpha=1.265$ : (a) $\mathrm{x}_{75}$; (b) $\ddot{\mathrm{x}}_{75}$; and (c) control force 
Table 2. A comparison of evaluation criteria with SMC (Wu, Yang 2004)

\begin{tabular}{|c|c|c|c|c|c|c|c|c|c|c|}
\hline \multicolumn{4}{|c|}{ Filter SMC $(\alpha=1.265, \tau=0.1)$} & \multicolumn{4}{|c|}{ Unfiltered SMC $(\alpha=1.265)$} & \multicolumn{3}{|c|}{ SMC (Wu, Yang 2004) } \\
\hline 莺 & $\begin{array}{l}0 \\
\| \\
\searrow\end{array}$ & $\begin{array}{l}\stackrel{\circ}{i} \\
\stackrel{y}{\sharp} \\
\frac{y}{y}\end{array}$ & 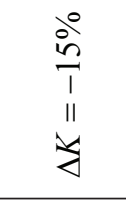 & 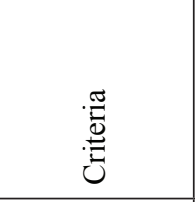 & $\begin{array}{l}0 \\
\| \\
\searrow\end{array}$ & $\begin{array}{l}i^{\circ} \\
\text { II } \\
i\end{array}$ & 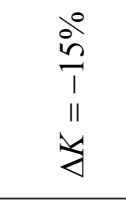 & 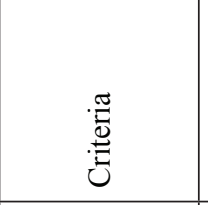 & $\begin{array}{l}0 \\
\| \\
\searrow \\
\searrow\end{array}$ & $\begin{array}{l}\stackrel{0}{i} \\
\frac{1}{\|} \\
\grave{y}\end{array}$ \\
\hline$J_{1}$ & 0.359 & 0.367 & 0.368 & $J_{1}$ & 0.353 & 0.351 & 0.371 & $J_{1}$ & 0.354 & 0.351 \\
\hline$J_{2}$ & 0.407 & 0.414 & 0.417 & $J_{2}$ & 0.400 & 0.397 & 0.420 & $J_{2}$ & 0.401 & 0.397 \\
\hline$J_{3}$ & 0.571 & 0.489 & 0.694 & $J_{3}$ & 0.569 & 0.482 & 0.700 & $J_{3}$ & 0.569 & 0.482 \\
\hline$J_{4}$ & 0.573 & 0.491 & 0.696 & $J_{4}$ & 0.571 & 0.484 & 0.702 & $J_{4}$ & 0.571 & 0.484 \\
\hline$J_{5}$ & 2.370 & 1.930 & 2.835 & $J_{5}$ & 2.436 & 1.964 & 2.944 & $J_{5}$ & 2.439 & 1.962 \\
\hline$J_{6}$ & 13.650 & 10.216 & 18.865 & $J_{6}$ & 14.715 & 10.626 & 20.525 & $J_{6}$ & 14.762 & 10.586 \\
\hline$\sigma_{u}(\mathrm{kN})$ & 39.00 & 32.86 & 50.52 & $\sigma_{u}(\mathrm{kN})$ & 39.90 & 33.51 & 51.99 & $\sigma_{u}(\mathrm{kN})$ & 40.07 & 33.55 \\
\hline$\sigma_{x_{m}(\mathrm{~cm})}$ & 24.03 & 19.565 & 28.74 & $\sigma_{x_{m}}(\mathrm{~cm})$ & 24.70 & 19.91 & 29.84 & $\sigma_{x_{m}}(\mathrm{~cm})$ & 24.73 & 19.89 \\
\hline$J_{7}$ & 0.364 & 0.420 & 0.464 & $J_{7}$ & 0.368 & 0.387 & 0.466 & $J_{7}$ & 0.369 & 0.387 \\
\hline$J_{8}$ & 0.424 & 0.455 & 0.524 & $J_{8}$ & 0.428 & 0.437 & 0.527 & $J_{8}$ & 0.428 & 0.437 \\
\hline$J_{9}$ & 0.703 & 0.624 & 0.767 & $J_{9}$ & 0.710 & 0.614 & 0.745 & $J_{9}$ & 0.711 & 0.613 \\
\hline$J_{10}$ & 0.711 & 0.631 & 0.776 & $J_{10}$ & 0.718 & 0.621 & 0.754 & $J_{10}$ & 0.719 & 0.620 \\
\hline$J_{11}$ & 2.338 & 1.963 & 3.011 & $J_{11}$ & 2.431 & 1.958 & 3.017 & $J_{11}$ & 2.436 & 1.958 \\
\hline$J_{12}$ & 75.664 & 61.346 & 137.350 & $J_{12}$ & 85.011 & 62.509 & 141.061 & $J_{12}$ & 85.644 & 62.453 \\
\hline $\max |u|(\mathrm{kN})$ & 140.55 & 129.47 & 189.61 & $\max |u|(\mathrm{kN})$ & 145.63 & 129.19 & 192.39 & $\max |u|(\mathrm{kN})$ & 145.98 & 129.00 \\
\hline $\max \left|x_{m}\right|(\mathrm{cm}) \mid$ & 75.50 & 63.41 & 97.24 & $\max \left|x_{m}\right|(\mathrm{cm})$ & 78.52 & 63.25 & 97.45 & $\max \left|x_{m}\right|(\mathrm{cm}) \mid$ & 78.68 & 63.24 \\
\hline
\end{tabular}

\section{Conclusions}

A filtered SMC approach is presented in the article for active vibration control of wind-excited highrise building structures and its performance is evaluated by application to a 76-story building benchmark problem equipped with an ATMD on the roof. Assuming rigid floors the 77-degree-of-fredom (DOF) structure is reduced to a $12-\mathrm{DOF}$ model. An asymptotic observer is employed to estimate the system states since only 3 out of the 12 states are measured directly. Compared with LQG and another implementation of SMC (Yang et al. 2004; Wu, Yang 2004), the proposed filtered SMC has in general better performance, especially in reducing the maximum control force and control power.

Innovations of the research can be summarized as follows:
1) The paper presents a new filtered sliding mode control (SMC) approach for vibration control of windexcited highrise building structures. Rather than using a Lyapunov-function based control design, an alternative way is provided to find the control force based on the equivalent control force principle to obtain the control force.

2) The main advantage of the SMC is that it is invariant to external excitation such as wind and earthquake and the variation of system parameters (such as structural stiffness and damping) during the dynamic event. The structural uncertainties can be represented by a linear combination of the control forces. However, the chattering in SMC is generally a problem that needs to be resolved for better control. To overcome this problem, a time-varying 
method is proposed for determining the sliding gain function in the SMC.

3) The performance of the proposed filtered SMC is evaluated by application to a large and real-life structural control problem, wind-excited 76-story building benchmark problem equipped with an active tuned mass damper (ATMD) on the roof.

4) The required control forces are reduced which means a reduction in the size of actuators, thus making the implementation of the new control algorithm more practical.

5) It is shown the proposed method is more robust to structural stiffness uncertainties and uncertainties compared with the linear quadratic Gaussian (LQG) algorithm and another implementation of SMC.

The proposed control algorithm is more robust than traditional LQG and unfiltered SMC. A second order filter may improve the results but not always. Further research includes the consideration of the actuator dynamics and actuator-structure interaction for practical implementation.

\section{References}

Adeli, H.; Gere, J.; Weaver, W., Jr. 1978. Algorithms for nonlinear structural dynamics, Journal of Structural Division 104(ST2): 263-280.

Adeli, H.; Jiang, X. 2009. Intelligent infrastructure: neural networks, wavelets, and Chaos theory for intelligent transportation systems and smart structures. Boca Raton, Florida: CRC Press, Taylor \& Francis. 440 p.

Adeli, H.; Kim, H. 2004. Wavelet-hybrid feedback-least mean square algorithm for robust control of structures, Journal of Structural Engineering 130(1): 128-137.

http://dx.doi.org/10.1061/(ASCE)0733-9445(2004)130:1(128)

Adeli, H.; Kim, H. 2009. Wavelet-based vibration control of smart building and bridge structures under earthquakes and winds. Boca Raton, Florida: CRC Press, Taylor \& Francis. 238 p.

Adeli, H.; Saleh, A. 1999. Control, optimization, and smart structures: High-performance bridges and buildings of the future. New York: John Wiley and Sons. 288 p.

Amini, F.; Khanmohamadi Hazaveh, N.; Abdolahi Rad, A. 2013. Wavelet PSO-based LQR algorithm for optimal structural control using active tuned mass dampers, Computer-Aided Civil and Infrastructure Engineering 28(7): 542-557. http://dx.doi.org/10.1111/mice.12017

Amini, F.; Zabihi-Samani, M. 2014. A wavelet-based adaptive pole assignment method for structural control, ComputerAided Civil and Infrastructure Engineering 29(6): 464477. http://dx.doi.org/10.1111/mice.12072

Boutalis, Y.; Christodoulou, M.; Theodoridis, D. 2013. Indirect adaptive control of nonlinear systems based on bilinear neuro-fuzzy approximation, International Journal of Neural Systems 23(5), 1350022.

http://dx.doi.org/10.1142/S0129065713500226

Davison, E. J. 1966. A method for simplifying linear dynamic systems, IEEE Transaction on Automatic Control 11(1): 93-101. http://dx.doi.org/10.1109/TAC.1966.1098264

El-Khoury, O.; Adeli, H. 2013. Recent advances on vibration control of structures under dynamic loading, Archives of Computational Methods in Engineering 20(4): 353-360. http://dx.doi.org/10.1007/s11831-013-9088-2

Fisco, N. R.; Adeli, H. 2011a. Smart structures: part I - active and semi-active control, Scientia Iranica - Transaction A: Civil Engineering 18(3): 275-284.
Fisco, N. R.; Adeli, H. 2011b. Smart structures: Part II - hybrid control systems and control strategies, Scientia Iranica Transaction A: Civil Engineering 18(3): 285-295.

Jiang, X.; Adeli, H. 2008a. Dynamic fuzzy wavelet neuroemulator for nonlinear control of irregular highrise building structures, International Journal for Numerical Methods in Engineering 74(7): 1045-1066.

http://dx.doi.org/10.1002/nme.2195

Jiang, X.; Adeli, H. 2008b. Neuro-genetic algorithm for nonlinear active control of highrise buildings, International Journal for Numerical Methods in Engineering 75(7): 770-786. http://dx.doi.org/10.1002/nme.2274

Kim, H.; Adeli, H. 2004. Hybrid feedback-least mean square algorithm for structural control, Journal of Structural Engineering 130(1): 120-127. http://dx.doi.org/10.1061/ (ASCE)0733-9445(2004)130:1(120)

Kim, H.; Adeli, H. 2005. Wind-induced motion control of 76-story benchmark building using the hybrid damperTLCD system, Journal of Structural Engineering 131(12): 1794-1802. http://dx.doi.org/10.1061/(ASCE)07339445(2005)131:12(1794)

Nigdeli, S. M.; Boduroğlu, M. H. 2013. Active tendon control of torsionally irregular structures under near-fault ground motion excitation, Computer-Aided Civil and Infrastructure Engineering 28(9): 718-736. http://dx.doi.org/10.1111/mice.12046

Rigatos, G. G. 2013. Adaptive fuzzy control for differentially flat MIMO nonlinear dynamical systems, Integrated Computer-Aided Engineering 20(2): 111-126.

Saleh, A.; Adeli, H. 1996. Parallel eigenvalue algorithms for large-scale control-optimization problems, Journal of Aerospace Engineering 9(3): 70-79. http://dx.doi.org/10.1061/(ASCE)0893-1321(1996)9:3(70)

Saleh, A.; Adeli, H. 1997. Robust parallel algorithms for solution of the Riccati equation, Journal of Aerospace Engineering 10(3): 126-133. http://dx.doi.org/10.1061/ (ASCE)0893-1321(1997)10:3(126)

Skelton, R. E.; Ikeda, M. 1989. Covariance controllers for linear continuous-time systems, International Journal of Control 49(5): 1773-1785. http://dx.doi.org/10.1080/00207178908559737

Smith, B. S.; Coull, A. 1992. Tall building structures: analysis and design. New York: Wiley. 537 p.

Su, W. C.; Huang, C. S.; Chen, C. H.; Liu, C. Y.; Huang, H. C.; Le, Q. T. 2014. Identifying the modal parameters of a structure from ambient vibration data via the stationary wavelet packet, Computer-Aided Civil and Infrastructure Engineering 29(10): 738-757.

http://dx.doi.org/10.1111/mice.12115

Tolu, S.; Vanegas, M.; Garrido, J. A.; Luque, N. R.; Ros, E. 2013. Adaptive and predictive control of a simulated robot arm, International Journal of Neural Systems 23(3), 1350010. http://dx.doi.org/10.1142/S012906571350010X

Utkin, V. I. 1993. Sliding mode control design principles and applications to electric drives, IEEE Transactions on Industrial Electronics 40(1): 23-36. http://dx.doi.org/10.1109/41.184818

Wu, J. C. 2003. Experiments on a full-scale building model using modified sliding model control, Journal of Engineering Mechanics 129(4): 363-372.

http://dx.doi.org/10.1061/(ASCE)0733-9399(2003)129:4(363)

Wu, J. C.; Yang, J. N. 2004. Modified sliding mode control for wind-excited benchmark problem, Journal of Engineering Mechanics 130(4): 499-504. http://dx.doi.org/10.1061/ (ASCE)0733-9399(2004)130:4(499)

Yang, J. N.; Wu, J. C.; Agrawal, A. K. 1995. Sliding mode control for seismically excited linear structures, Journal of Engineering Mechanics 121(12): 1386-1390. http://dx.doi.org/10.1061/(ASCE)0733-9399(1995) 121:12(1386) 
Yang, J. N.; Agrawal, A.; Samali, B.; Wu, J. C. 2004. A benchmark problem for response control of wind excited tall buildings, Journal of Engineering Mechanics 130(4): 437-446. http://dx.doi.org/10.1061/(ASCE)07339399(2004)130:4(437)

\section{APPENDIX I}

\section{Explanation of formulation of the benchmark problem}

In Eqn (19), $m=500$ metric ton, and $k_{m}=505.1 \mathrm{kN} / \mathrm{m}$ are the mass, damping coefficient, and stiffness value of the ATMD, respectively. For the coupled ATMD-structure system, Eqns (1) and (19) are combined and an equation similar to Eqn (1) is found where $\boldsymbol{M}, \boldsymbol{C}, \boldsymbol{K}, \boldsymbol{\eta}$, and $\boldsymbol{B}_{s}$ are replaced by the following matrices:

$\boldsymbol{M}_{c}=\left[\begin{array}{cc}\boldsymbol{M} & 0 \\ \boldsymbol{a} & m\end{array}\right] \in \boldsymbol{R}^{77 \times 77}$ where $\boldsymbol{a}=\left[\begin{array}{llll}0 & \cdots & 0 & m\end{array}\right] \in \boldsymbol{R}^{76}$, $\boldsymbol{C}_{c}=\left[\begin{array}{cc}\boldsymbol{C} & 0 \\ 0 & c_{m}\end{array}\right] \in \boldsymbol{R}^{77 \times 77}, \quad \boldsymbol{K}_{c}=\left[\begin{array}{cc}\boldsymbol{K} & 0 \\ 0 & k_{m}\end{array}\right] \in \boldsymbol{R}^{77 \times 77}$, $\boldsymbol{\eta}_{c}=\left[\begin{array}{ll}\boldsymbol{\eta} & 0 \\ 0 & 0\end{array}\right] \in \boldsymbol{R}^{77 \times 77}, \quad \boldsymbol{B}_{c}=\left[\begin{array}{llll}0 & \cdots & 0 & 1\end{array}\right]^{\boldsymbol{T}} \in \boldsymbol{R}^{77}$, respectively. The displacement vector is also expanded to $\boldsymbol{x}=\left[x_{1}, x_{2}, \ldots, x_{76}, x_{m}\right]^{T}$ where $x_{i}$ is the displacement of the $i$ th floor and $x_{m}$ is the displacement of the ATMD relative to the top floor.

The parameters in the LQG control approach of the benchmark problem are given as: $\mathbf{Q}=\operatorname{diag}[1,1,1,1,1,1,1,1$, $\left.1,0,1,1,1,1,1,10^{5}, 10^{5}, 10^{5}, 10^{5}, 0,1,1,1,1,1,10^{5}, 10^{5}, 10^{5}, 10^{5}, 0\right]$, where $\operatorname{diag}[]$ is used here to represent a diagonal matrix in which the entries outside the main diagonal are all zeros. $\mathrm{R}=0.1, \mathrm{~S}_{\boldsymbol{v}_{\boldsymbol{r}} \boldsymbol{v}_{\boldsymbol{r}}}(\omega)=\operatorname{diag}[0.1,0.1,0.1]$. For simplicity $\boldsymbol{S}_{\boldsymbol{w} w}$ is scaled as $\left.S_{w w}(\omega)=1.524 \times 10^{4} \times\left|\overline{\boldsymbol{W}}(\omega) \overline{\boldsymbol{W}}^{\boldsymbol{T}}(\omega)\right|\right)$ at $\omega=0.1743 \mathrm{rad} / \mathrm{s}$ that achieves a peak value of

$\boldsymbol{S}_{w \boldsymbol{w}}(\omega)$. The term $\overline{\boldsymbol{W}}(\omega)$ is the Fourier transform of the wind load $\boldsymbol{W}(t)$. The control output vector is $\boldsymbol{Z}=[\boldsymbol{z}, \dot{\boldsymbol{z}}, \ddot{z}]^{T} \in \boldsymbol{R}^{30}$ where

$$
\boldsymbol{z}=\left[x_{1}, x_{30}, x_{50}, x_{55}, x_{60}, x_{65}, x_{70}, x_{75}, x_{76}, x_{m}\right] \in \boldsymbol{R}^{10} .
$$

Therefore, the weight matrix is $\mathbf{Q} \in R^{30}$ rather than $R^{12}$. The weight $\mathrm{R}$ is for the control force. For the purpose of practical implementation, the following hardware limitations are chosen for the actuator: the peak control input/ force $|u|_{\max } \leq 300 \mathrm{kN}$, RMS value of the control input (related to energy consumption) $\sigma_{u} \leq 100 \mathrm{kN}$, peak relative displacement of the ATMD $\left|x_{m}\right|_{\max } \leq 95 \mathrm{~cm}$, and RMS value of the relative displacement of the ATMD $\sigma_{x m} \leq 30 \mathrm{~cm}$.

\section{APPENDIX II}

\section{Performance Criteria for the 76-story benchmark problem}

The evaluation criteria fall into three categories: peak responses, normalized responses, and control requirements. A total of 16 criteria are provided for evaluation of the performance of a control algorithm (Yang et al. 2004). They are summarized in this Appendix.

The maximum root mean squared (RMS) value of acceleration:

$J_{1}=\max \left\{\sigma_{\ddot{x}_{1}}, \sigma_{\ddot{x}_{30}}, \sigma_{\ddot{x}_{50}}, \sigma_{\ddot{x}_{55}}, \sigma_{\ddot{x}_{60}}, \sigma_{\ddot{x}_{65}}, \sigma_{\ddot{x}_{70}}, \sigma_{\ddot{x}_{75}}\right\} / \sigma_{\ddot{x}_{750}}$,

where $\sigma_{\ddot{x}_{i}}$ is the RMS value of acceleration of the $i$ th floor, $\sigma_{\ddot{x}_{750}}=9.142 \mathrm{~cm} / \mathrm{s}^{2}$ is the RMS value of acceleration of the $75^{\text {th }}$ floor without control.

The average acceleration of 6 selected floors above the $49^{\text {th }}$ floor:

$$
J_{2}=\frac{1}{6} \sum_{i} \frac{\sigma_{\ddot{x}_{i}}}{\sigma_{\ddot{x}_{i o}}}
$$

where $i=50,55,60,65,70$, and 76 , and $\sigma_{\ddot{x}_{i o}}$ is the RMS value of acceleration of the $i$ th floor without control.

The ratio of the displacements of top floor with and without control:

$$
J_{3}=\frac{\sigma_{x_{76}}}{\sigma_{x_{76 o}}},
$$

where $\sigma_{x_{76}}$ and $\sigma_{x_{760}}$ are the RMS values of the displacement of the $76^{\text {th }}$ floor with and without control, respectively $\left(\sigma_{x_{760}}=10.137 \mathrm{~cm}\right)$.

The average ratio of the displacements of 7 selected floors with and without control:

$$
J_{4}=\frac{1}{7} \sum_{i} \frac{\sigma_{x_{i}}}{\sigma_{x_{i o}}},
$$

where $i=50,55,60,65,70,75,76$, and $\sigma_{x_{i}}$ and $\sigma_{x_{i o}}$ is the RMS value of the displacement of the $i$ th floor with and without control, respectively.

The nondimensionalized actuator stroke (displacement) and average power:

$$
\begin{gathered}
J_{5}=\frac{\sigma_{x_{m}}}{\sigma_{x_{76 o}}} ; \\
J_{6}=\sigma_{P}=\sqrt{\frac{1}{T} \int_{0}^{T}\left[\dot{x}_{m}(t) u(t)\right]^{2} d t},
\end{gathered}
$$

where $\sigma_{x_{m}}=$ RMS of the actuator stroke, $\dot{x}_{m}(t)=$ actuator velocity, $T=$ total time of integration (chosen as 900 seconds), and $\sigma_{P}=$ RMS of the control power. 
Criteria $J_{7}$ to $J_{10}$ are defined in terms of maximum structural response as follows:

$$
\begin{gathered}
J_{7}=\max \left\{\ddot{x}_{p 1}, \ddot{x}_{p 30}, \ddot{x}_{p 50}, \ddot{x}_{p 55}, \ddot{x}_{p 60}, \ddot{x}_{p 65}, \ddot{x}_{p 70}, \ddot{x}_{p 75}\right\} / \ddot{x}_{p 750} ; \\
J_{8}=\frac{1}{6} \sum_{i} \frac{\ddot{x}_{p i}}{\ddot{x}_{p i o}},
\end{gathered}
$$

for $i=50,55,60,65,70,75$, and

$$
\begin{gathered}
J_{9}=\frac{x_{p 76}}{x_{p 76 o}} ; \\
J_{10}=\frac{1}{7} \sum_{i} \frac{x_{p i}}{x_{p i o}},
\end{gathered}
$$

for $i=50,55,60,65,70,75,76$, where $x_{p i}$ and $x_{p i o}$ are the peak displacements of the $i$ th floor with and without control, respectively; $\ddot{x}_{p i}$ and $\ddot{x}_{p i o}$ are the peak accelerations of the $i$ th floor with and without control, respectively; $x_{p 76 o}=32.30 \mathrm{~cm}$ and $\ddot{x}_{p 76 o}=32.33 \mathrm{~cm} / \mathrm{s}^{2}$.

The following criteria are proposed for evaluation of the required actuators:

$$
J_{11}=\frac{x_{p m}}{x_{p 76 o}}
$$

$$
J_{12}=P_{\max }=\max \left|\dot{x}_{m}(t) u(t)\right|,
$$

where $x_{p m}=$ peak actuator stroke, and $P_{\max }=$ peak control power.

The remaining four (unnumbered) are the RMS value of the control force $\left(\sigma_{u}\right)$, the RMS value of the actuator stroke $\left(\sigma_{x_{m}}\right)$, the absolute maximum value of the control force $(\max |u|)$, and the absolute maximum value of the actuator stroke $\left(\max \left|x_{m}\right|\right)$.

Nengmou WANG. He is a graduate student at the Ohio State University. His areas of research interests include building design, sustainability, and vibration control. He has published several papers in Journal of the Franklin Institute and Computer Applications in Engineering Education.

Hojjat ADELI. He is a Professor of Civil, Environmental, and Geodetic Engineering at the Ohio State University. He has authored/ co-authored over 270 journal articles in various fields of computer science, engineering, mathematics, and medicine since 1976 when he received his $\mathrm{PhD}$ from Stanford University at the age of 26 . He has authored/co-authored 15 books. In 1995 , he wrote Machine Learning - Neural Networks, Genetic Algorithms, and Fuzzy Sets (published by John Wiley and Sons), the first authored book that covers and integrates the three main areas of soft computing. He also holds a United States patent for his neural dynamic computational model. He is the quadruple winner of The Ohio State University Lumley Outstanding Research Award. In 1998 he received the Ohio State University's highest research honor, the Distinguished Scholar Award "in recognition of extraordinary accomplishment in research and scholarship". In 2005, he was elected Distinguished Member, American Society of Civil Engineers. In 2007, he received the Ohio State University College of Engineering Peter L. and Clara M. Scott Award for Excellence in Engineering Education as well as the Charles E. MacQuigg Outstanding Teaching Award. In 2008, he was elected a Fellow of the American Association for the Advancement of Science (AAAS). He was elected a Fellow of IEEE in 2012. He is the Editor-in-Chief of the international research journal Computer-Aided Civil and Infrastructure Engineering which he founded in 1986. 